\title{
Parenting and the Emotional and Behavioural Adjustment of Young Children in Families with a Parent with Bipolar Disorder
}

\author{
Rachel Calam \\ University of Manchester, $U K$ \\ Steven Jones \\ Lancaster University, $U K$ \\ Matthew R. Sanders \\ University of Queensland, Australia \\ Robert Dempsey and Vaneeta Sadhnani \\ University of Manchester, UK
}

\begin{abstract}
Background: Children of parents with bipolar disorder are at increased risk of disturbance. Aims: This study examined relationships between parental mood, parenting, household organization and child emotional and behavioural adjustment in families with a parent with bipolar disorder to determine areas of specific need for parenting support. Method: 48 parents were recruited through advertisements via self-help organizations. The study was conducted online. Parental mood and activity was assessed by self-report questionnaires (CES-D, ISS, MDQ and SRM); parenting was assessed using the Parenting Scale (PS). The SDQ was used to assess the parent's view of their child's emotional and behavioural difficulties. The Confusion, Hubbub and Order Scale (CHAOS) assessed household organization. Results: Parents reported high levels of difficulties across all measures and scores were above clinical cut-offs on most scales. Children were reported as showing high levels of disturbance on the SDQ, including all sub-scales. Parenting and depression scores were significantly positively correlated, as were depression, parenting and CHAOS score. Regression analyses indicated that CHAOS was the strongest predictor of Total Difficulties and Emotional Symptoms on the SDQ. Conclusions: Families are likely to benefit from interventions tailored to meet their parenting needs.
\end{abstract}

Reprint requests to Rachel Calam, School of Psychological Sciences, University of Manchester, Zochonis Building, Brunswick Street, Manchester M13 9PL, UK. E-mail: rachel.calam@manchester.ac.uk 
Keywords: Child anxiety, child behaviour problems, childhood anxiety, children, family, hypomania, depression, parent training, parental behaviour.

\section{Introduction}

Children growing up in families with a parent with bipolar disorder are at increased risk of a range of behavioural and emotional difficulties including depression and bipolar disorder (Duffy, Alda, Crawford, Milin and Grof, 2007; Hillegers et al., 2005; Jones, Tai, Evershed, Knowles and Bentall, 2006; Reichart et al., 2004). Early identification and intervention is indicated, to reduce the risk of mood disorders in this group. Furthermore, parenting difficulties in themselves may represent significant stressors, with the potential to further exacerbate bipolar symptoms in the symptomatic parent. Intervention to help parents manage parenting difficulties more effectively ought to help the adjustment of the parent with bipolar disorder, while at the same time reducing difficulty and distress for other family members. In order to design the best interventions it is important to understand the nature of the difficulties that family members experience, and inter-relationships between these.

Literature on the children of parents with bipolar disorder indicates elevated levels of emotional and behavioural difficulties (Birmaher et al., 2009; Jones and Bentall, 2008). Prospective studies indicate that, by adolescence, $40-50 \%$ of children of bipolar parents will develop a mood disorder, and 10-20\% bipolar disorder (Duffy et al., 2007; Hillegers et al., 2005). There are also indications that attentional and behavioural difficulties are particularly associated with risk (Carlson and Weintraub, 1993). Research indicates more negative communication styles in bipolar parents compared to controls (Vance, Huntley Jones, Espie, Bentall and Tai, 2008) and parents with other, non-bipolar, psychopathology (Inoff-Germain, Nottelmann and Radke-Yarrow, 1992).

Child behavioural difficulties exist in the context of negative coercive cycles of interaction (Reid and Patterson, 1989), which also contribute to negative mood states in the parent. There is an extensive literature on the reciprocal relationships between maternal depression and behavioural and emotional difficulties in children (Shaw, Connell, Dishion, Wilson and Gardner, 2009). Some specific parenting behaviours associated with parental mood, including laxness, verbosity and over-reactivity, are associated with increased risk of conduct problems in children (O'Leary, Slep and Reid, 1999). Parenting interventions to improve child behaviour have the potential to intervene in negative cycles of coercive interaction that contribute to disorder in both the child and the parent. Given the predominance of depressive symptoms in the long term course of bipolar disorder, improvements in such negative cycles (Judd et al., 2003) would offer the potential to break into processes that may contribute to intergenerational transmission of disorder.

A further predictor of disorder in the children of parents with bipolar disorder may be household organization. Reports (Ostiguy et al., 2008) indicate high levels of stressors in the lives of these offspring and the degree of instability found in their homes (Chang, Blasey, Ketter and Steiner, 2001; Romero, Delbello, Soutullo, Stanford and Strakowski, 2005). Instability and lack of organization in the household has been captured in other literature by the construct of chaos (Matheny and Wachs, 1995), which is linked to parenting (Corapci and Wachs, 2002). This dimension provides an important additional predictor for the development of children's problem behaviour (Coldwell, Pike and Dunn, 2006). 
This preliminary study examined ratings of difficulties in households where there was a parent with bipolar disorder in order to examine relationships between variables, and to build theory in the area so as to develop the potential for models for intervention. We examined parental mental health variables relevant to both bipolar disorder and parenting, including: mood disorder; depression; activation; perceived conflict and well being; self-ratings of parenting, including laxness, verbosity, over-reactivity; parent ratings of child adjustment, and household chaos.

We predicted that where parents were more depressed, they would report greater laxness in parenting. We predicted that higher levels of activation, characterized by systemic arousal, restlessness and impulsivity, would be associated with higher levels of parental over-reactivity and verbosity. Assuming that high levels of activation would lead to unpredictability and inconsistency in parenting, we hypothesized that where parents were more unstable, their children would be reported as showing higher levels of behavioural difficulty. We also predicted that higher activation would be associated with greater levels of household chaos. We predicted that when parents were more depressed, showing lower levels of well-being, their children would show higher levels of emotional difficulties.

\section{Method}

Ethical approval for the study was granted by the University's Senate Ethics Committee.

\section{Participants}

Fifty-two parents responded to advertisements for the study and completed questionnaires online. Parents and caregivers of 33 girls and 29 boys registered for the study. Due to noncompletion of some questionnaires, only 48 families are described here. Forty-five $(93.8 \%)$ participants were mothers, two (4.2\%), fathers, and one, a stepmother. Parent characteristics are shown in Table 1. The children had a mean age of 6.0 years $(2 \mathrm{yr}$ minimum, $10 \mathrm{yr}$ maximum); there were 29 boys (60.4\%) and 19 girls (39.6). No inclusion or exclusion criteria were applied other than age of child. If parents had more than one child, they were asked to choose one child within the age range and confine their responses to that child.

\section{Measures}

Demographic data were collected using questions used in previous online parenting research with a UK sample (Sanders, Calam, Durand, Liversidge and Carmont, 2008).

Mood Disorders Questionnaire (MDQ; Hirschfeld et al., 2000). The MDQ was developed to aid diagnosis of bipolar disorders. In the original version it covers symptom endorsement of 13 manic/hypomanic symptoms (Section 1), symptom clustering (Section 2) and severity of problem caused (Section 3). Research supports the use of Section 1 alone to avoid incorrect screening out of individuals experiencing bipolar II symptoms (Twiss, Jones and Anderson, 2008). This research indicated that the optimal cut-off score, 9 or more, endorsed symptoms without applying the supplementary questions (sensitivity of 0.90 and 0.88 for bipolar I and bipolar II groups respectively with a specificity of 0.90 ). 
Table 1. Participant demographics

\begin{tabular}{lcl}
\hline Variable & Number $(\%)$ & \multicolumn{1}{c}{ Description } \\
\hline Family composition* & $24(50.0)$ & Original family (biological or adoptive) \\
& $6(12.5)$ & Step family \\
& $15(31.3)$ & Single parent family \\
Participant's highest level of education & $2(4.2)$ & Other (extended family) \\
& $10(20.8)$ & No qualifications \\
& $4(8.3)$ & GCSEs/O-Levels \\
& $3(6.3)$ & A Levels/BTEC \\
Employment and benefits & $15(31.3)$ & University degree /apprenticeships \\
& $14(29.2)$ & Postgraduate qualification \\
& $31(64.6)$ & Currently employed \\
Finances & $17(35.4)$ & Not employed \\
& $11(22.9)$ & Currently on benefits \\
Ethnicity & $37(77.1)$ & Not on benefits \\
& $19(39.6)$ & Good finances \\
& $25(51.2)$ & Managing to get by \\
& $4(8.3)$ & Struggling \\
& $38(79.2)$ & $78 \%$ White \\
& $8(16.7)$ & $16 \%$ Mixed \\
& $1(2.1)$ & $2 \%$ Black-African \\
$1(2.1)$ & $2 \%$ Chinese \\
& $1(2.1)$ & $2 \%$ Other \\
\hline
\end{tabular}

* Not reported by one family

The Center for Epidemiological Studies Depression Scale (CES-D; Radloff, 1991). CES-D is a 20-item self-report depression scale that detects current depressive symptoms. A score above 16 indicates possible depression (Radloff, 1991).

Internal States Scale (ISS; Bauer et al., 1991). This is a self-report questionnaire assessing manic and depressive symptoms. Its 15 items contribute to four subscales: Activation (ISSA), Well-Being (ISS-WB), Perceived Conflict (ISS-PC), and Depression (ISS-D). Each item relates to the preceding 24 hours. Ratings are made on a $100 \mathrm{~mm}$ visual analogue scale with anchor descriptions of "Not at all/Rarely" at one extreme and "Very much so/Much of the time" at the other. This measure is widely used and has good internal reliability and validity. An ISS-A score above 200 indicates possible mania; ISS-WB below 125 is indicative of possible depression (Bauer et al., 1991).

Social Rhythm Metric (SRM; Monk, Flaherty, Frank, Hoskinson and Kupfer, 1990). Stability of activity patterns was assessed by means of SRM (Monk et al., 1990), which assesses the importance of zeitgebers (cues that set the biological clock, including social cues such as work schedules, regularity of meal routines, and timing of social contacts) and zeitstorers (events that disrupt circadian rhythms; for example, having a young child that does not sleep; being in a very stressful work environment with no "down time"; breaking-up with a long term partner) in BD (Frank, Swartz and Kupfer, 2000). Event-reporting rates did not differ significantly over 12 weeks in a study comparing depressed and control participants (Monk et al., 1990). A trait version of SRM has been developed in which each item is rated 
for the previous month. If an event regularly occurs within 45 min on at least 3 days per week, a rating is obtained for the number of days [3-7]. Two scores are obtained: SRM total (number of events that occur regularly) and SRM average (average frequency for regular events) (Shen, Alloy, Abramson and Sylvia, 2008; Shen, Sylvia et al., 2008).

Strengths and Difficulties Questionnaire (SDQ; Goodman, 2001). This is a widely used, 25-item questionnaire with good psychometric properties (Goodman, 2001) generating dimensional scores for total difficulties, emotional symptoms, conduct problems, hyperactivity, peer problems and prosocial behaviour. The measure is capable of identifying children with depressive, conduct and hyperactive disorders. It has shown a specificity of 94.6\% (95\% CI 94.1-95.1\%) and sensitivity of 63.3\% (59.7-66.9\%) in identifying individuals with a psychiatric diagnosis (Goodman, Ford, Simmons, Gatward and Meltzer, 2000).

The Parenting Scale (PS; Arnold, O'Leary, Wolff and Acker, 1993). This 30-item questionnaire measures dysfunctional discipline styles in parents. Test-retest reliabilities for the three subscales were .83 (Laxness), .82 (Overreactivity) and .79 (Verbosity). It reliably discriminates parents of clinical and non-clinical children (Arnold et al., 1993).

Confusion, Order and Hubbub Scale (CHAOS; Matheny and Wachs, 1995). The short 6item version of this scale was used. This includes items such as "you can't hear yourself think in our home" and "the atmosphere in our house is calm" rated on a 5-point scale.

\section{Procedure}

Parents of 2- to 10-year-old children were invited to participate in the study via publicity in newsletters, email circulars and at local meetings of the MDF (The Bipolar Organization - MDF). A weblink for the study was included in all advertising. All questionnaires were completed online. The online participant information explained that the survey would give either immediate or deferred access to a trial of a web-based parenting intervention.

\section{Statistical analysis}

Data were analyzed using SPSS version 16. Pearson correlations were used to examine relationships between variables. Linear multiple regression with stepwise backward entry was used to test the contribution of variables to child behaviour, with forward entry to confirm the final model.

\section{Results}

Half $(50 \%)$ of the children were living in their original biological or adoptive family. The majority of participating parents had studied to degree level, and $29 \%$ had a postgraduate qualification. The majority (65\%) were currently employed. Only $8 \%$ of the sample described themselves as struggling financially.

\section{Participants}

Eight $(16.7 \%)$ of the children were in contact with a professional over concerns. Of these, four parents indicated that these were in relation to anxiety and negative feelings, ADHD/mood disorder or behaviour problems; one cited enuresis, and others gave less specific descriptions 
Table 2. Parental contact with health professionals in the last 6 months

\begin{tabular}{lll}
\hline & \multicolumn{1}{c}{ Self } & Partner \\
\hline & YES & YES \\
Psychologist & $15(31.3)$ & $2(4.2)$ \\
Psychiatrist & $39(81.2)$ & $2(4.2)$ \\
Counsellor & $15(31.2)$ & $6(12.5)$ \\
Social Worker & $12(25.0)$ & $1(2.1)$ \\
Other & $25(52.1)$ & $6(12.5)$ \\
\hline
\end{tabular}

or were awaiting their appointment. Two parents were contemplating seeking help. A far higher percentage of the parents had themselves been in contact with professionals over their own difficulties (Table 2).

\section{Levels of difficulties}

Table 3 shows the levels of difficulties reported on the questionnaire measures, together with published, established clinical cut-off scores for scales, where available.

Child emotional and behavioural difficulties. Table 3 shows the percentage of children in the borderline and abnormal categories on the SDQ. For comparison, approximately $80 \%$ of children would be expected to be in the normal range of scores, $10 \%$ borderline and $10 \%$ abnormal (Goodman, 2001). A much higher percentage of the current sample showed borderline or abnormal adjustment on all scales than would be expected.

Parental adjustment. The mean score for the sample on the MDQ was 12.4, and all parents scored above the cut-off of 9, suggesting the presence of bipolar disorder (Table 3 ). The mean score on the CES-D, 36.5, was markedly higher than the cut-off for depression of 16 for the scale. The mean ISS activation score, 183.2, was below the cut-off; scores below 200 are not indicative of current mania. In contrast, the ISS-WB mean score of 106.5, again below cut-off (125), indicates possible depression.

Using these indicators, the sample appeared to be within the clinical range in terms of lifetime bipolar status and current depression. On the Social Rhythm Metric, the number of regular activities was just above the cut-off of 9.9, but the frequency with which individuals engaged in these activities was lower than expected, with a mean of 3.1 compared to the cut-off of 5.

Parenting practices. Scores on the Parenting Scale indicated that families had high levels of dysfunctional parenting, compared to the mean total score of $3.6(S D=0.8)$ found in the clinical sample used in the original development study (Arnold et al., 1993).

CHAOS. The mean score of 17.4 for this sample is much higher than a score of 2.43 for a sample of 118 UK households (Coldwell et al., 2006).

Depression, parenting and chaos. Pearson correlations indicated that CES-D total score was significantly correlated with total score on the Parenting Scale $(r=0.309, p=.033)$ and with the overreactivity subscale $(r=0.304 p=.035)$. The other sub-scales of the Parenting Scale, laxness and verbosity, did not show significant correlations $(r=0.198$, laxness; $r=$ 0.114, verbosity). Parents who reported higher CES-D scores also had higher scores on the 
Table 3. Child adjustment, parental mood and parenting scores and clinical cut-offs $(N=48)$

\begin{tabular}{|c|c|c|c|}
\hline \multirow[b]{2}{*}{$\begin{array}{l}\text { SDQ emotional } \\
\text { symptoms }\end{array}$} & \multirow{2}{*}{$\frac{\text { Mean }(S D)}{4.0(2.5)}$} & \multicolumn{2}{|c|}{$\begin{array}{l}\text { Clinical cut-offs ( } \% \text { of } \\
\text { sample in range, SDQ) }\end{array}$} \\
\hline & & $\begin{array}{l}4 \\
(18.8)^{1}\end{array}$ & $\begin{array}{l}5-10 \\
(39.6)^{2}\end{array}$ \\
\hline SDQ conduct problems & $4.4(2.6)$ & $\begin{array}{l}3 \\
(10.4)^{1}\end{array}$ & $\begin{array}{l}4-10 \\
(60.4)^{2}\end{array}$ \\
\hline SDQ hyperactivity & $5.8(2.4)$ & $\begin{array}{l}6 \\
(12.5)^{1}\end{array}$ & $\begin{array}{l}7-10 \\
(43.8)^{2}\end{array}$ \\
\hline SDQ peer problems & $3.0(1.9)$ & $\begin{array}{l}3 \\
(18.8)^{1}\end{array}$ & $\begin{array}{l}4-10 \\
(39.6)^{2}\end{array}$ \\
\hline SDQ prosocial & $6.0(2.2)$ & $\begin{array}{l}5 \\
(6.8 \%)^{1}\end{array}$ & $\begin{array}{l}0-4 \\
(34.1 \%)^{2}\end{array}$ \\
\hline SDQ total difficulties & $17.2(6.1)$ & $\begin{array}{l}14-16 \\
(12.5)^{1}\end{array}$ & $\begin{array}{l}17-40 \\
(60.4)^{2}\end{array}$ \\
\hline Depression (CES-D) & $36.5(16.9)$ & \multicolumn{2}{|c|}{$>16$ depression } \\
\hline ISS activation & $183.2(124.1)$ & \multicolumn{2}{|c|}{$>200$ mania } \\
\hline ISS depression & $126.4(150.6)$ & & \\
\hline ISS perceived conflict & $223.5(112.8)$ & \multirow{3}{*}{\multicolumn{2}{|c|}{$\begin{array}{l}<125 \text { depression } \\
>9 \text { bipolar disorder }\end{array}$}} \\
\hline ISS well-being & $106.5(75.7)$ & & \\
\hline Mood disorder Q (MDQ) & $12.4(1.0)$ & & \\
\hline *Social rhythm metric (SRM) & $9.9(3.2)$ & & \\
\hline Parenting Scale (PS) total & $3.6(0.8)$ & & \\
\hline PS laxness & $3.2(01.0)$ & & \\
\hline PS overreactivity & $3.6(1.1)$ & & \\
\hline PS verbosity & $4.0(0.8)$ & & \\
\hline Confusion, Hubbub, and Order Scale (CHAOS) & $17.4(5.1)$ & & \\
\hline
\end{tabular}

${ }^{1}$ cut-off, borderline, SDQ ${ }^{2}$ cut-off, abnormal, SDQ

CHAOS $(r=.328, p=.023)$, perhaps indicating more general difficulties with household management.

Activation, parenting and chaos. We found no support for our prediction that higher levels of activation would be associated with higher levels of parental over-reactivity $(r=0.102$, $\mathrm{ns})$ and verbosity ( $r=-0.056, \mathrm{~ns})$. ISS activation showed no significant correlation with the either the total score $(r=0.167, \mathrm{~ns})$ or the laxness sub-scale $(r=0.234, \mathrm{~ns})$ on the Parenting Scale. The correlation between ISS activation and CHAOS was non-significant $(r=0.200$, ns).

Activation and adjustment in the child. There was a significant positive correlation between ISS activation and emotional difficulties in the child $(r=0.348, p=0.016)$. There were no significant correlations with parental report of other difficulties in the child, either for the total SDQ score $(r=0.140, \mathrm{~ns})$ or any of the other SDQ subscales (range, Pearson correlations, $r=0.003-0.120, \mathrm{~ns})$.

Parental mood and child emotional adjustment. Non-significant correlations indicated no support for the hypothesis that when parents showed lower levels of well-being on the CES-D, their children would show higher levels of emotional difficulties.

Household organization, parenting and child adjustment. There was support for the prediction that there would be higher levels of difficulties in parenting and child adjustment 
Table 4 Final model, multiple regression with forward entry, CES total, CHAOS and emotional symptoms on the SDQ

\begin{tabular}{lccc}
\hline & B & SE B & beta \\
\hline Constant & 0.88 & 1.20 & \\
CHAOS total & 0.18 & 0.07 & $.37^{*}$ \\
\hline
\end{tabular}

${ }^{*} p=.009$

where there were higher levels of disorganization. The CHAOS showed significant positive correlations with overreactivity on the Parenting Scale $(r=.384, p=.007)$ and with the child adjustment outcomes of emotional symptoms $(r=.374, p=.009)$ and total score on the SDQ $(r=.301, p=.038)$.

\section{Regression analyses}

Total score on the SDQ. Linear multiple regression with stepwise backward entry was used to test the contributions of CES-D Total, CHAOS and overreactivity to the total score for difficulties reported on the SDQ. The regression equation only became significant when CHAOS was the sole predictor of SDQ total $(F=4.58, d f 1,46, p=.038, B=.36, S E .17$, beta $=.301)$. This pattern was confirmed by repeating the regression omitting overreactivity, using forward entry, and using only CES-D and CHAOS; CES-D was excluded and only CHAOS remained in the model $(F=7.49$, df $1,46, p=.009, R=.374, R$ square $=.140)$. Table 4 shows this analysis.

\section{Discussion}

This preliminary study examined difficulties experienced by parents with bipolar disorder and the emotional and behavioural adjustment of their children to identify whether they have particular needs for parenting support. Parents rated themselves as having both considerable personal difficulties and difficulties in raising their children, and the majority described their children as having high levels of difficulties in adjustment. Very few were currently engaged with a professional because of their child's difficulties.

The sample was small, and self-selected from parents involved with self-help groups for bipolar disorder, and all measures were self-reported by a single informant, in almost all cases the mother, so we have to be tentative in our conclusions. The sample was also relatively affluent and well educated so it is not clear therefore to what extent the findings would generalize more broadly. The online format will have engaged computer-literate parents with computers in their own homes or workplace. However it is unlikely that the level of problems observed in this sample would be lower in a sample of parents with fewer financial advantages.

Emotional difficulties often precede the development of later psychopathology in families with a parent with bipolar disorder (Duffy, 2009). There is accumulating evidence that an episode of depression can be a first indicator for the development of bipolar disorder, as demonstrated by three longitudinal cohort studies (Duffy et al., 2007; Hillegers et al., 2005; Reichart et al., 2004). However, other emotional difficulties including anxiety may be 
prodromal (Duffy et al., 2007; Shaw, Gilliom, Ingoldsby and Nagin, 2003). In the present study, the emotional symptoms sub-scale of the SDQ gave indications of the presence of high levels of anxiety and depression in the majority of the children in the sample. For the conduct problems subscale, the mean was within the abnormal range. For hyperactivity, again, a very high percentage of the children were reported as showing significant difficulties. This is also noteworthy, as ADHD has been found to be a risk factor for bipolar disorder in retrospective adult samples (Sachs, Baldassano, Truman and Guille, 2000) and questionnaire ratings of child behaviour indicating ADHD are predictive in prospective, longitudinal samples (Biederman et al., 2009).

For peer problems, the majority of children had borderline or abnormal ratings, and the percentage showing low levels of prosocial behaviour was higher than would be expected. While other studies of children of bipolar parents have shown that adolescent offspring do not necessarily show impairment in social functioning (Ostiguy et al., 2008; Reichart et al., 2004), our findings indicate that, for this sample at least, parents reported considerable difficulties. Scores may well reflect sampling; parents particularly concerned about child behaviour and emotional adjustment may have volunteered. The findings do, however, make clear that there is a population of bipolar parents who find their children's adjustment problematic.

Unlike many studies of depressed parents, there was little indication in our sample that parental depression was directly positively correlated with ratings of child behaviour. This may indicate a ceiling effect, for this small, self-selected sample. It is also noteworthy that we did not find the relationships we had predicted between scores on the Parenting Scale and ratings of child emotional and behavioural difficulty. The reported measurements were taken at a single time point; a larger scale, longitudinal study with measurement of fluctuations in parental mood, symptoms and parenting over time would give a more accurate picture of relationships between parental disorder and child outcomes, particularly given the cyclical nature of bipolar disorder.

It was, however, clear that parental mental health was associated with parenting overall, as measured by the Parenting Scale, particularly the Overreactivity sub-scale. There was the predicted coherence in the associations found between the ratings that parents gave of their own mental state and their parenting, but this did not relate directly to the levels of difficulties that they reported experiencing with their children. Parents reporting higher levels of activation were also more likely to report emotional difficulties in their children. There were associations between parental mental state, household chaos and child emotional adjustment. Regression analyses indicated that CHAOS ratings were most strongly predictive of high scores for emotional difficulties in the child. These are cross-sectional analyses, so causality cannot be inferred.

Considering the relevance of these findings to intervention, there is accumulating evidence for the important role of environmental aspects on developmental trajectory in offspring of parents with bipolar disorder. One review (DelBello and Geller, 2001) draws attention to aspects of the environment that appear to influence the development of the children of bipolar parents. Having a healthy father may reduce the risk of developing symptoms (Conrad and Hammen, 1993). Negative reactions of mothers with bipolar disorder to their children indicates that quality of parenting might be a significant contributor to outcome (InoffGermain et al., 1992). Duffy et al. (2007) found children of bipolar parents who were well and asymptomatic in their childhood and early- and mid-adolescent years continued to show good adjustment. 
Our findings indicate that high levels of overreactivity, of activation and chaos in the household are particularly associated with emotional difficulties in the child. We do not yet know whether early intervention to improve parenting could create an environment in which children can develop free from symptoms, and whether engaging a non-symptomatic parent may be particularly helpful. The evidence, however, that the development of significant behavioural difficulties in children is preventable, with appropriate intervention (Zubrick et al., 2005) has the potential to make a significant contribution to establishing the necessary conditions for a healthier developmental trajectory for high-risk children. Heterogeneity of course may be a significant aspect to consider; for example, children of parents who are lithium non-responders may have a different trajectory (Duffy et al., 2007). Studies testing the impact of interventions to improve parenting and family environment, taking detailed account of parental characteristics, would be illuminating.

Work on stress in the family environment (Ostiguy et al., 2008), and the literature indicating that the family environment created by parents with bipolar disorder tends towards instability also suggests potential value for intervention. In our study, parents who were more depressed were more likely to describe their household as chaotic; higher scores on the CHAOS were also associated with greater parenting difficulties, in particular over-reactivity. Higher CHAOS scores were also associated with higher ratings of child emotional difficulties. Our sample size was insufficient to pursue analyses to indicate the extent to which household chaos might mediate relationships, but it does suggest that helping parents to establish a more ordered, organized and predictable household would be an important element of an evidence-based parenting intervention tailored to the needs of families with a parent with bipolar disorder.

The majority of parents were already in contact with professionals on their own behalf. Where parents have mental health difficulties and are already accessing services, the additional element of intervention for their child's behaviour and adjustment represents a significant burden. It is important to find ways of offering minimally sufficient, effective parenting interventions in a format that can be used flexibly alongside other forms of professional input, so that parents can implement new strategies whenever they can make time to do so. There is a developing body of research that aims to identify the best ways of providing parenting support to parents with severe mental illness which acknowledges the centrality of parenting to emotional well-being and self-esteem, and the therapeutic importance of recognizing parenting concerns (David, Styron and Davidson, 2011).

Taken together, our findings and the research literature to date suggest a number of elements that would be valuable in intervention. Developing a more stable and predictable environment for the child is likely to be valuable, alongside reducing negative parental reactions to the child and improving quality of parenting. Helping parents develop strategies to reduce overreactivity is likely to be important. High quality evidence-based parenting interventions have the potential to improve these, particularly where they are designed to address parental difficulties alongside management of the child. For example, Enhanced Level 5 Triple P (Sanders, Bor and Morawska, 2007) combines strategies to improve parenting with integrated intervention to address other parental issues.

Furthermore, the difficulties parents with mental health difficulties face in finding time and space for an intervention concerning their child alongside their own needs may require modification to methods of delivery. Parents experience practical demands on time, due to their own need for engagement with services for their own mental health, and personal difficulties arising from their own mental health needs. These may restrain parents from 
seeking help at a point that is appropriate for their child. There may also be practical constraints arising from the availability of appropriate integrated services in their locality. Self-directed, web-based approaches (Calam, Sanders, Miller, Sadhnani and Carmont, 2008; Sanders et al., 2008) and telephone support could provide opportunities for parents who might not otherwise be able to make time in the working day to access services. Further studies aimed at increasing our understanding of the parenting needs and best interventions for families where there is a parent with significant mental health difficulties have the potential to prevent or significantly reduce mental health risks and improve developmental trajectory for vulnerable children.

\section{References}

Arnold, D. S., O'Leary, S. G., Wolff, L. and Acker, M. M. (1993). The Parenting Scale: a measure of dysfunctional parenting in discipline situations. Psychological Assessment, 5, 137-144.

Bauer, M. S., Crits-Christoph, P., Ball, W. A., Dewees, E., McAllister, T., Alahi, P., et al. (1991). Independent assessment of manic and depressive symptoms by self-rating: scale characteristics and implications for the study of mania. Archives of General Psychiatry, 48, 807-812.

Biederman, J., Petty, C. R., Monuteaux, M. C., Evans, M., Parcell, T., Faraone, S. V., et al. (2009). The Child Behavior Checklist-Pediatric Bipolar Disorder profile predicts a subsequent diagnosis of bipolar disorder and associated impairments in ADHD youth growing up: a longitudinal analysis. Journal of Clinical Psychiatry, 70, 732-740.

Birmaher, B., Axelson, D., Monk, K., Kalas, C., Goldstein, B., Hickey, M. B., et al. (2009). Lifetime psychiatric disorders in school-aged offspring of parents with bipolar disorder: the Pittsburgh Bipolar Offspring Study. Archives of General Psychiatry, 66, 287-296.

Calam, R., Sanders, M. R., Miller, C., Sadhnani, V. and Carmont, S. A. (2008). Can technology and the media help reduce dysfunctional parenting and increase engagement with preventative parenting interventions? Child Maltreatment, 13, 347-361.

Carlson, G. A. and Weintraub, S. (1993). Childhood behavior problems and bipolar disorderrelationship or coincidence? Journal of Affective Disorders, 28, 143-153.

Chang, K. D., Blasey, C., Ketter, T. A. and Steiner, H. (2001). Family environment of children and adolescents with bipolar parents. Bipolar Disorder, 3, 73-78.

Coldwell, J., Pike, A. and Dunn, J. (2006). Household chaos: links with parenting and child behaviour. Journal of Child Psychology and Psychiatry, 47, 1116-1122.

Conrad, M. and Hammen, C. (1993). Protective and resource factors in high and low-risk children: a comparison of children with unipolar, bipolar, medically ill, and normal mothers. Development and Psychopathology, 5, 593-607.

Corapci, F. and Wachs, T. D. (2002). Does parental mood or efficacy mediate the influence of environmental chaos upon parenting behavior? Merrill-Palmer Quarterly, 48, 182-201.

David, D. H., Styron, T. and Davidson, L. (2011). Supported parenting to meet the needs and concerns of mothers with severe mental illness. American Journal of Psychiatric Rehabilitation, 14, 137-153.

DelBello, M. P. and Geller, B. (2001). Review of studies of child and adolescent offspring of bipolar parents. Bipolar Disorder, 3, 325-334.

Duffy, A. (2009). The early course of bipolar disorder in youth at familial risk. Journal of the Canadian Academy of Child and Adolescent Psychiatry, 18, 200-205.

Duffy, A., Alda, M., Crawford, L., Milin, R. and Grof, P. (2007). The early manifestations of bipolar disorder: a longitudinal prospective study of the offspring of bipolar parents. Bipolar Disorder, 9 , 828-838. 
Frank, E., Swartz, H. A. and Kupfer, D. J. (2000). Interpersonal and social rhythm therapy: managing the chaos of bipolar disorder. Biological Psychiatry, 48, 593-604.

Goodman, R. (2001). Psychometric properties of the strengths and difficulties questionnaire. Journal of the American Academy of Child and Adolescent Psychiatry, 40, 1337-1345.

Goodman, R., Ford, T., Simmons, H., Gatward, R. and Meltzer, H. (2000). Using the Strengths and Difficulties Questionnaire (SDQ) to screen for child psychiatric disorders in a community sample. British Journal of Psychiatry, 177, 534-539.

Hillegers, M. H., Reichart, C. G., Wals, M., Verhulst, F. C., Ormel, J. and Nolen, W. A. (2005). Five-year prospective outcome of psychopathology in the adolescent offspring of bipolar parents. Bipolar Disorder, 7, 344-350.

Hirschfeld, R. M. A., Williams, J. B. W., Spitzer, R. L., Calabrese, J. R., Flynn, L., Keck Jr., P. E., et al. (2000). Development and validation of a screening instrument for bipolar: the Mood Disorder Questionnaire. American Journal of Psychiatry, 157, 1873-1875.

Inoff-Germain, G., Nottelmann, E. D. and Radke-Yarrow, M. (1992). Evaluative communications between affectively ill and well mothers and their children. Journal of Abnormal Child Psychology, $20,189-212$.

Jones, S. H. and Bentall, R. P. (2008). A review of potential cognitive and environmental risk markers in children of bipolar parents. Clinical Psychology Review, 28, 1083-1095.

Jones, S. H., Tai, S., Evershed, K., Knowles, R. and Bentall, R. (2006). Early detection of bipolar disorder: a pilot familial high-risk study of parents with bipolar disorder and their adolescent children. Bipolar Disorders, 8, 362-372.

Judd, L. L., Akiskal, H. S., Schettler, P. J., Coryell, W., Maser, J., Rice, J. A., et al. (2003). The comparative clinical phenotype and long term longitudinal episode course of bipolar I and II: a clinical spectrum or distinct disorders? Journal of Affective Disorders, 73, 19-32.

Matheny, A. P. and Wachs, T. D. (1995). Bringing order out of chaos: psychometric characteristics of the Confusion, Hubbub and Order Scale. Journal of Applied Developmental Psychology, 16, 429-444.

Monk, T. H., Flaherty, J. F., Frank, E., Hoskinson, K. and Kupfer, D. J. (1990). The Social Rhythm Metric: an instrument to quantify the daily rhythms of life. Journal of Nervous Mental Disorders, $178,120-126$.

O'Leary, S. G., Slep, A. M. and Reid, M. J. (1999). A longitudinal study of mothers' overreactive discipline and toddlers' externalizing behavior. Journal of Abnormal Child Psychology, 27, 331-341.

Ostiguy, C. S., Ellenbogen, M. A., Linnen, A. M., Walker, E. F., Hammen, C. and Hodgins, S. (2008). Chronic stress and stressful life events in the offspring of parents with bipolar disorder. Journal of Affective Disorders, 114, 74-84.

Radloff, L. S. (1991). The use of the Centre for Epidemiologic Studies Depression Scale in adolescents and young adults. Journal of Youth and Adolescence, 20, 149-166.

Reichart, C. G., Wals, M., Hillegers, M. H., Ormel, J., Nolen, W. A. and Verhulst, F. C. (2004). Psychopathology in the adolescent offspring of bipolar parents. Journal of Affective Disorders, 78, 67-71.

Reid, J. B. and Patterson, G. R. (1989). The development of antisocial behaviour patterns in childhood and adolescence. European Journal of Personality, 3, 107-119.

Romero, S., Delbello, M. P., Soutullo, C. A., Stanford, K. and Strakowski, S. M. (2005). Family environment in families with versus families without parental bipolar disorder: a preliminary comparison study. Bipolar Disorder, 7, 617-622.

Sachs, G. S., Baldassano, C. F., Truman, C. J. and Guille, C. (2000). Comorbidity of attention deficit hyperactivity disorder with early- and late-onset bipolar disorder. American Journal of Psychiatry, 157, 466-468.

Sanders, M. R., Bor, W. and Morawska, A. (2007). Maintenance of treatment gains: a comparison of enhanced, standard, and self-directed Triple P-Positive Parenting Program. Journal of Abnormal Child Psychology, 35, 983-998. 
Sanders, M. R., Calam, R., Durand, M., Liversidge, T. and Carmont, S. A. (2008). Does selfdirected and web-based support for parents enhance the effects of viewing a reality television series based on the Triple P-Positive Parenting Programme? Journal of Child Psychology and Psychiatry, 49, 924-932.

Shaw, D. S., Connell, A., Dishion, T. J., Wilson, M. N. and Gardner, F. (2009). Improvements in maternal depression as a mediator of intervention effects on early childhood problem behavior. Development and Psychopathology, 21, 417-439.

Shaw, D. S., Gilliom, M., Ingoldsby, E. M. and Nagin, D. S. (2003). Trajectories leading to school-age conduct problems. Developmental Psychology, 39, 189-200.

Shen, G. H., Alloy, L. B., Abramson, L. Y. and Sylvia, L. G. (2008). Social rhythm regularity and the onset of affective episodes in bipolar spectrum individuals. Bipolar Disorder, 10, 520-529.

Shen, G. H., Sylvia, L. G., Alloy, L. B., Barrett, F., Kohner, M., Iacoviello, B., et al. (2008). Lifestyle regularity and cyclothymic symptomatology. Journal of Clinical Psychology, 64, 482-500.

Twiss, J., Jones, S. and Anderson, I. (2008). Validation of the Mood Disorder Questionnaire for screening for bipolar disorder in a UK sample. Journal of Affective Disorders, 110, 180-184.

Vance, Y. H., Huntley Jones, S., Espie, J., Bentall, R. and Tai, S. (2008). Parental communication style and family relationships in children of bipolar parents. British Journal of Clinical Psychology, 47, 355-359.

Zubrick, S. R., Ward, K. A., Silburn, S. R., Lawrence, D., Williams, A. A., Blair, E., et al. (2005). Prevention of child behavior problems through universal implementation of a group behavioral family intervention. Prevention Science, 6, 287-304. 